\title{
A New Approach: Quantitative Imaging Spectrometry for Iron Oxide Analysis
}

\author{
Jeffrey M. Davis ${ }^{1}$, Julia Schmidt ${ }^{1}$, Martin Huth $^{1}$, Robert Hartmann ${ }^{2}$, Heike Soltau ${ }^{1}$, Lothar Strüder ${ }^{2,3}$ \\ 1. PNDetector GmbH, Otto-Hahn-Ring 6, 81739 München, Germany \\ 2. PNSensor GmbH, Otto-Hahn-Ring 6, 81739 München, Germany \\ 3. University of Siegen, Walter-Flex-Strasse 3, 57072 Siegen, Germany
}

Solid state X-ray detectors have undergone major improvements and advances in the last 50 years. Such advances include, but are not limited to, improvements in energy resolution, count rate and solid angle. One significant improvement is the development of detectors capable of recording both the position and energy of every X-ray event that hits the detector. Such detectors are known as imaging spectrometers, since they combine the spectroscopic performance of the silicon drift detector with the position resolution of an imaging detector.

The Color X-ray Camera (CXC) uses an imaging spectrometer known as the pnCCD [1]. This unique device delivers count rates of up to $1 \mathrm{Mcps}$ with an energy resolution of $150 \mathrm{eV}$ at the $\mathrm{Mn} \mathrm{K} \alpha$ line. With these performance characteristics, the CXC enables simultaneous X-ray diffraction (XRD) and X-ray fluorescence (XRF) experiments [2]. Using an imaging spectrometer to collect both the XRD and XRF signals eliminates the need to make a $\theta-2 \theta$ scan, thereby decreasing the time required to collect a diffraction pattern. Furthermore, the position resolution of the detector allows for the separation of the diffraction and fluorescence signals, resulting in background free diffraction patterns and fluorescence spectra without diffraction peaks [2].

To highlight a practical application of quantitative imaging spectrometry, a series of iron oxide samples were measured in the forward scattering (Bragg-Brentano) geometry. The iron oxide samples were taken from various stages in an iron ore refinement process, where the goal was to reduce hematite into wuestite and refine silica out of the stock iron ore. Figure 1 shows a typical diffraction pattern and fluorescence spectrum from one of the samples. One typical problem with the XRD analysis of iron oxide is the copious fluorescence signal that the material will create. This results in a much lower peak to background ratio in the diffraction pattern, requiring longer scans in traditional XRD instruments. Through the use of an imaging spectrometer, the signals can be readily separated. The presence of diffraction peaks in X-ray fluorescence spectra is also difficult to remove with traditional XRF techniques and instruments. Diffraction peaks are typically elimited by filtering the primary X-ray beam, but this will reduce the intensity of the primary beam, necessitating longer measurement times. Again, through the separation of the signal, the true fluorescence spectrum can be recovered. Figure 2 shows a plot of the calcium content of the iron oxide samples, determined by both fluorescence and diffraction methods. Calcite is added to the iron oxide to reduce the silicon content. When heated, the calcite decomposes into free lime $(\mathrm{CaO})$ and carbon dioxide. The free lime combines with silicon present in natural iron oxide and becomes fayalitic limestone slag, which is later removed. By tracking the calcium content using the diffraction peaks from calcite and the $\mathrm{Ca} \mathrm{K} \alpha$ line, the conversion from calcite to free lime can be identified, as shown in Figure 2. This unique method for interpreting data was acquired from a single measurement, in a single instrument with no moving parts. 
References:

[1] O Scharf et al, Analytical Chemistry 83 (2011), p. 2532.

[2] JM Davis et al, Proceedings of Microscopy and Microanalysis (2016), p. 102.
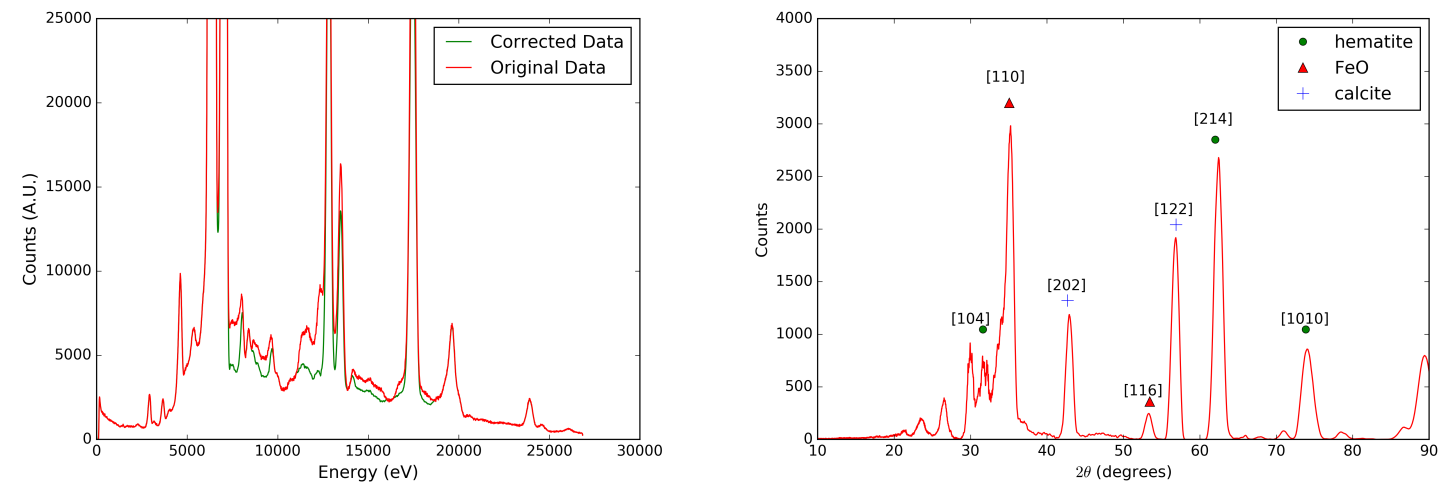

Figure 1: Two spectra derived from a simultaneous XRD-XRF analysis of an iron oxide powder sample. (Left) The corrected (green) and uncorrected (red) fluorescence spectrum. Since the vast majority of the signal comes from the $\mathrm{Fe} \mathrm{K} \alpha, \beta$ lines, the diffraction peaks are not easy to identify from simple peak shapes. (Right) The diffraction pattern showing the three major phases identified in the material.

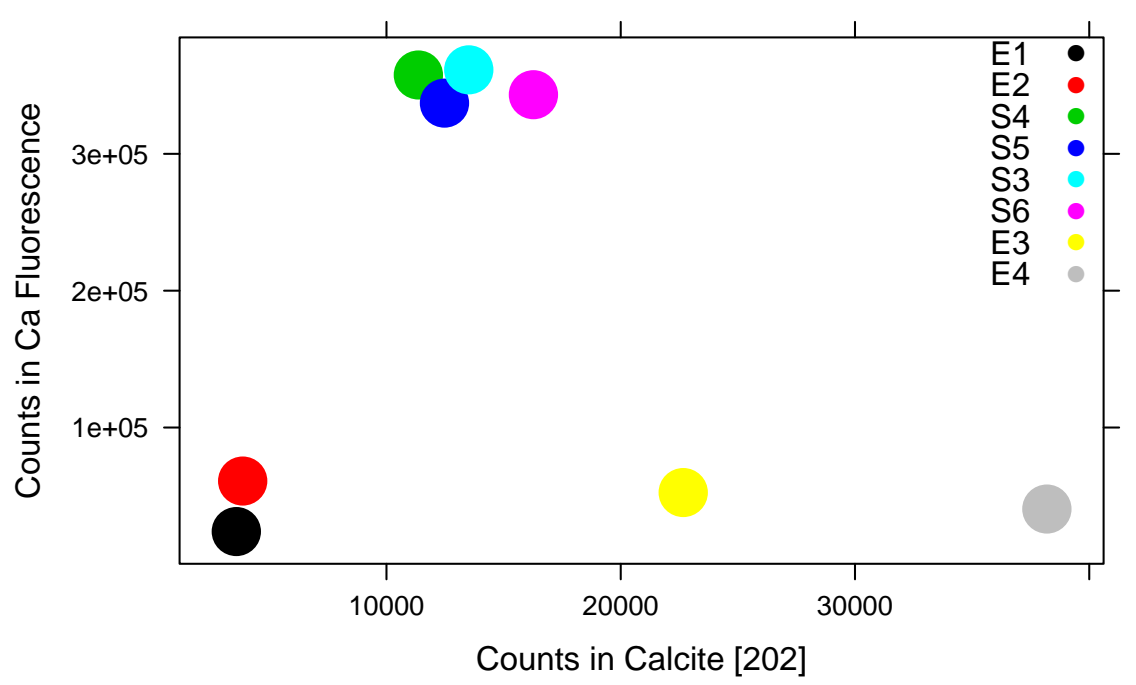

Figure 2: A plot showing the counts in the Calcite [202] diffraction peak versus the counts in the $\mathrm{Ca} \mathrm{K} \alpha$ fluorescence peak for eight iron oxide samples. Samples E3 and E4 (bottom right of the graph) show that most of the calcium present in those samples is calcite. The cluster of points called S3, S4, S5 and S6 shows a middle phase where some of the calcium is in calcite, but much of the signal comes from other calcium phases (likely free lime, $\mathrm{CaO}$ ). Observing this transition is important for the online quality control in steel fabrication. 